\title{
Hepatitis C y VIH en usuarios de drogas inyectables en Armenia-Colombia
}

\section{Hepatitis C and HIV in injecting drug \\ users in Armenia, Colombia}

\author{
Dedsy Berbesi-Fernández, Ángela Segura-Cardona, Liliana Montoya-Vélez, Guillermo A. \\ Castaño-Perez*
}

*Docentes-Investigadores, Facultad de Medicina. Universidad CES

\section{Resumen}

Un aumento constante y progresivo en la disponibilidad de heroína en Colombia en las últimas décadas, y el uso de esta droga vía intravenosa, ha establecido la necesidad de prevenir una posible epidemia de VIH y hepatitis C. Esta investigación determino la seroprevalencia de hepatitis C y VIH según características sociodemográficas y comportamientos de riesgo en población que se inyecta drogas en Armenia-Colombia. Estudio transversal en 265 usuarios captados a través de muestreo guiado por el encuestado previo consentimiento informado. La seroprevalencia de hepatitis C fue 22,3\% [IC95\% 12,3\%-23,5\%]; la infección por VIH fue 2,6\% [IC95\% 0,4-6,0]. El 67,5\% reportó inyección por más de dos años, el $35 \%$ compartió jeringas y agujas y el 12,4\% utilizó condón en su última relación sexual. Los usuarios que no adquirían jeringas en droguerías en los últimos seis meses tienen 2,7 [IC95\% 1,32-5,48] veces el riesgo de hepatitis C; la frecuencia diaria de inyección fue mayor en los casos positivos con VIH [OR 2,87; IC95\% 0,55-15,9] pero no significativa. La cuarta parte de los encuestados, están infectados por VIH o hepatitis C, en forma de infección única o coinfección. Este estudio identificó prácticas de riesgo, como compartir jeringas y baja utilización del condón en los últimos seis meses, factores de riesgo documentados y discutidos mundialmente. Esta investigación constituye un primer paso en la búsqueda de estrategias para prevenir la propagación de infecciones por $\mathrm{VIH}$ y hepatitis $\mathrm{C}$ en redes de usuarios de drogas inyectables.

Palabras clave: hepatitis C, VIH, heroína, epidemiologia.

\begin{abstract}
A constant and progressive increase in the availability of heroin in Colombia in recent decades and the intravenous use of this drug have established the need to prevent a possible epidemic of HIV and hepatitis C. This research determined the sero-prevalence of hepatitis $\mathrm{C}$ and HIV according to sociodemographic characteristics and risk behaviors in people who inject drugs in Armenia, Colombia. This is a cross-sectional study on 265 users captured through respondentdriven sampling after informed consent. Sero-prevalence of hepatitis C was $22.3 \%$ [95\% CI $12.3 \%-23.5 \%$ ]; for HIV infection, it was $2.6 \%$ [95\% CI 0.4 to 6.0 ]; $67.5 \%$ reported injecting for more than two years, $35 \%$ shared needles and syringes, and $12.4 \%$ had used a condom during their last sexual intercourse. Users who did not purchase syringes in drugstores in the last six months are 2.7 times [95\% CI 1.32 to 5.48] more likely to contract hepatitis C; daily injection frequency was higher in HIV- positive cases [OR 2.87; 95\% CI 0.55 to 15.9] but nonsignificant. One fourth of respondents are infected with HIV or hepatitis C, either as a single infection or co-infection. This study identified risk practices such as sharing needles and low condom use in the last six months, worldwide documented and discussed risk factors. This research is a first step in the search for strategies to prevent the spread of HIV infection and hepatitis C in networks of injecting drug users.

Key words: hepatitis C, HIV, heroin, epidemiology.
\end{abstract}




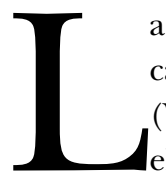

a Organización Mundial de la Salud calcula que cada año se infectan con el virus de la hepatitis $\mathrm{C}$ (VHC) entre tres y cuatro millones de personas en el mundo, 150 millones de personas sufren la infección crónica y 350.000 personas mueren por causa de enfermedades hepáticas vinculadas con la hepatitis (WHO, 2012)

La hepatitis C tiene una distribución mundial. Se calcula que cada usuario de drogas inyectables (UDI) que adquiere VHC puede transmitir el virus aproximadamente a 20 personas, y la mitad de estas transmisiones se producen en los primeros dos años de la infección (Magiorkinis et al., 2013).

Según ONUSIDA la transmisión del VIH por consumo de drogas inyectables continúa siendo uno de los desafíos más importantes; se estima que en 49 países la prevalencia de VIH en personas que se inyectan drogas es 22 veces mayor que en el resto de la población; y en 11 países el nivel de infección es de 50 veces superior. Se suma a este desafío el estigma y la falta de acceso a los servicios de salud (ONUSIDA, 2012).

La seropositividad por VIH en población de 15 a 49 años en el mundo, es de $0,8 \%$ pero la morbilidad por esta infección sigue variando considerablemente entre países y regiones (UNAIDS, 2012).

Las epidemias de VIH y VHC asociadas al uso inyectado de drogas han sido ampliamente documentadas y hoy, la búsqueda de una intervención adecuada y oportuna es una de las acciones prioritarias en países en los que se visibiliza este tipo de consumo (WHO, 2012).

Los países de América Latina se caracterizan por presentar una epidemia principalmente concentrada de VIH, afectando a personas que usan drogas, trabajadores(as) sexuales, personas transgénero y personas que practican sexo con hombres. Entre estas personas que usan drogas, el grupo que se ha identificado como más vulnerable es el de usuarios de drogas inyectables (Bravo \& Barrio, 2008).

En el 2010 se realizó un estudio de prevalencia de VIH y comportamientos de riesgo-en usuarios de drogas por vía inyectada (UDI) en otras dos ciudades de Colombia (Medellín y Pereira), cuyo resultado reportó prevalencias del 3,8\% y $1,9 \%$ respectivamente; ese estudio concluyó que existe riesgo de expansión de VIH a otras ciudades de Colombia entre las redes de personas que usan drogas inyectables (Berbesi et al., 2012). Como es de esperarse, en Colombia la infección por VIH también ha sido definida como una epidemia concentrada que cumple con las condiciones que establece la Organización Mundial de la Salud (ONUSIDA, 2000).

Durante las últimas décadas, un aumento constante y progresivo en la disponibilidad de heroína en Colombia, ha establecido la necesidad de prevenir una posible epidemia de infecciones como VIH y VHC debido al consumo de drogas por vía intravenosa (Berbesi et al., 2013); y aunque el consumo de drogas está despenalizado en el país desde 1994, no se identifica un desarrollo adecuado del sistema de salud, ni políticas claras frente a programas de mitigación, reducción o intercambio de jeringas (Gómez, 2013).
Por lo tanto, este estudio buscó estimar las prevalencias de VIH y VHC y la identificación de las conductas de riesgo para la transmisión entre usuarios de drogas inyectables en Armenia-Colombia.

\section{Método}

Se realizó un estudio transversal utilizando el muestreo guiado por el encuestado, la población objetivo fueron los usuarios de drogas inyectables residentes en la ciudad de Armenia ${ }^{1}$-Colombia, se consideraron como criterios de inclusión ser usuario activo de drogas inyectables (en los últimos seis meses); tener entre 18 y 65 años de edad, firmar el consentimiento informado y contar con un cupón vigente de RDS (Respondent Driven Sampling).

El reclutamiento se inició con un grupo de "semillas" o participantes seleccionados de forma no aleatoria de la población objetivo a través de informantes claves; a estas personas se les entregó dos tipos de incentivos económicos: primario por participar en el estudio y, secundario vinculado al reclutamiento exitoso de tres nuevos participantes; los valores de los incentivos variaron entre US\$ 5 y US\$ 4 respectivamente.

Para el cálculo del tamaño muestral, se utilizó la fórmula de Fleiss para estimar una proporción, el nivel de confianza fue $95 \%$, la precisión absoluta fue $4 \%$, efecto de diseño de 4 y con una proporción esperada del $3 \%$ teniendo en cuenta la prevalencia de VIH en la población UDI en el año 2010 (Berbesi et al., 2012).El tamaño de la muestra fue de 250 usuarios de drogas inyectables; el muestreo por RDS estima de manera propia los errores de muestreo a través de medidas como homofilia, heterofilia y estimaciones proporcionales de la población. El tamaño de muestra se alcanzó en un plazo de seis semanas.

Se utilizó el instrumento aplicado a población UDI de Medellín y Pereira en el año 2010 (Berbesi et al., 2012), el cual se basó en el realizado por la OMS en Bogotá en el año 2000 por Mejía \& Gómez (2005); este formulario incluyó preguntas acerca de las características demográficas y comportamientos de riesgo y fue aplicado por un grupo de entrevistadores previamente capacitado y estandarizado; además se tomó muestra de sangre para realizar prueba de laboratorio en papel de filtro para detección de VHC y VIH; los casos fueron confirmados por pruebas de Carga Viral de RNA y Western Blot respectivamente.

El análisis estadístico de los datos y las tablas de resultados se generaron mediante el RDSAT ${ }^{\circledR}$ y SPSS ${ }^{\circledR}$ 21.0. Se realizó la descripción de las características sociodemográficas de los UDI y la determinación de prevalencia de VHC

1 Armenia es considerada una ciudad mediana, que realiza funciones de intermediación entre núcleos más pequeños y las grandes áreas metropolitanas de país, su población proyectada para el 2014 es de aproximadamente 400.000 según cifras del Departamento Administrativo Nacional de Estadística (DANE). 
y VIH con sus intervalos de confianza del 95\% (IC 95\%). La asociación entre la frecuencia de inyección, compartir jeringas y agujas, compra de jeringas en la droguería y el uso del condón en los últimos seis meses con las prevalencias de VHC y VIH, se estableció a través de pruebas Chi-cuadrado y la fuerza de la asociación por medio OR e IC $95 \%$.

Las medidas de asociación crudas para VHC se ajustaron mediante regresión logística binaria, donde se incluyeron factores asociados o con valores $\mathrm{p}$ inferiores a 0,25 (criterio de Hosmer-Lemeshow), no se realizó este análisis para VIH debido al bajo número de casos.

Este proyecto fue avalado por el Comité de Institucional de Ética de la Universidad CES, se garantizó la confidencialidad y se tomó consentimiento informado.

\section{Resultados}

Con relación a las características sociodemográficas de los 265 usuarios de drogas inyectables que participaron del estudio, se encontró que el 87\% (214) eran de sexo masculino, el $76 \%$ eran solteros y $48 \%$ (136) se encontraban en el grupo etario de 25 a 34 años. La edad media fue de 26,8 (DE: 5,68) años; el rango de edad fue de 18 a 50 años. Por sexo, la media de edad para los hombres fue 27 años y para las mujeres fue 25,7 años. En términos de estrato socioeconómico, el 83,3\% eran de estrato bajo, un 14,4\% de estrato medio y de estrato alto el 2,3\%. El 75,8\% de los UDIs tenían secundaria completa, un $27,9 \%$ de los UDIs describieron como actividades para adquirir los ingresos ser independientes, un $25,3 \%$ realizaban trabajos temporales y un 20 , $8 \%$ refirieron ser vendedores ambulantes.
Tabla 1

Estimación proporcional de las características sociodemográficas de los usuarios de drogas inyectables. Armenia-Colombia.

\begin{tabular}{|c|c|c|}
\hline Grupo de edad & $\begin{array}{c}\text { Estimación } \\
\text { proporcional de la } \\
\text { población (\%) }\end{array}$ & IC $95 \%$ \\
\hline \multicolumn{3}{|l|}{ Edad } \\
\hline 18 a 24 años & 42,0 & $34,0-49,4$ \\
\hline 25 a 34 años & 48,0 & $40,7-56,1$ \\
\hline 35 a 44 años & 7,1 & $3,8-10,7$ \\
\hline 45 y más años & 2,9 & $0,7-5,8$ \\
\hline \multicolumn{3}{|l|}{ Sexo } \\
\hline Hombres & 87,3 & $82,9-92,8$ \\
\hline Mujeres & 12,7 & $7,2-17,1$ \\
\hline \multicolumn{3}{|l|}{ Nivel de escolaridad } \\
\hline Primaria & 11,4 & $6,7-16,3$ \\
\hline Secundaria & 75,8 & $68,7-82,1$ \\
\hline Superior & 12,4 & $7,7-18,5$ \\
\hline \multicolumn{3}{|l|}{ Estado civil } \\
\hline Soltero & 76,2 & $70,6-81,8$ \\
\hline Casado/Unión libre & 17,2 & $12,0-22,8$ \\
\hline Divorciado/Separado & 5,6 & $2,8-8,7$ \\
\hline Viudo & 1,0 & $0,0-2,8$ \\
\hline
\end{tabular}

Nota. *IC: Intervalos de confianza

Tabla 2

Características demográficas y comportamientos de riesgo en usuarios de drogas inyectables relacionados con Hepatitis $\mathrm{C}$ y VIH. Armenia 2014.

\begin{tabular}{|c|c|c|c|c|c|c|c|c|c|c|}
\hline \multirow[b]{2}{*}{ Variable } & \multicolumn{5}{|c|}{ Hepatitis C } & \multicolumn{5}{|c|}{ VIH } \\
\hline & $n$ & $\%$ & ORc & IC & Valor de $p$ & $\mathbf{n}$ & $\%$ & ORc & IC & Valor de $p$ \\
\hline \multicolumn{11}{|l|}{ Sexo } \\
\hline Mujeres & 8 & 22,9 & 1,03 & $0,44-2,40$ & 0,95 & 1 & 2,9 & 1,06 & $0,12-9,11$ & 0,96 \\
\hline Hombres & 50 & 22,4 & 1,00 & & & 6 & 2,7 & 1,00 & & \\
\hline \multicolumn{11}{|l|}{ Escolaridad } \\
\hline Primaria & 7 & 23,3 & 1,07 & $0,44-2,64$ & 0,88 & 2 & 6,7 & 3,29 & $0,61-17,7$ & 0,17 \\
\hline Secundaria & 52 & 22,1 & 1,00 & & & 5 & 2,1 & 1,00 & & \\
\hline \multicolumn{11}{|c|}{ Frecuencia de Inyección (últimos 6 meses) } \\
\hline Diario & 30 & 24,0 & 1,2 & $0,68-2,16$ & 0,52 & 5 & 4,0 & 2,87 & $0,55-15,9$ & 0,21 \\
\hline \multicolumn{11}{|c|}{ Comparte jeringas (ultimo seis meses) } \\
\hline $\mathrm{Si}$ & 22 & 24,2 & 1,18 & $0,64-2,16$ & 0,6 & 7 & 3,3 & 1,4 & $0,30-6,42$ & 0,66 \\
\hline No & 36 & 21,3 & 1,00 & & & 4 & 2,4 & 1,00 & & \\
\hline
\end{tabular}

Nota. *OR: Razón de disparidad 
Para la infección por VIH se registró una prevalencia de $2,6 \%$ (IC95\% 0,4-6,0) Se identificaron $1,1 \%$ de resultados indeterminados, que quizá responden a la etapa de seroconversión. Para hombres la prevalencia de VIH fue de 2,7\% y para las mujeres fue $2,9 \%$. No se identificó asociación estadísticamente significativa entre sexo y prevalencia de VIH.

Así mismo, el 31,0\% de los usuarios de drogas inyectables, presentó reactividad para anticuerpos del virus de hepatitis C (VHC), y un 22,3\% (IC 95\% 12,3\%-23,5\%) infección activa de hepatitis C. Por sexo, la prevalencia de hepatitis en los hombres fue de $22,4 \%$ y en las mujeres fue de $22,9 \%$, sin diferencias estadísticamente significativas.
Respecto a las prácticas de consumo investigadas el 67,5\% de los usuarios reportó haberse inyectado por más de dos años y un $35 \%$ ha compartido jeringas y agujas. En relación con las prácticas sexuales, el 87,6\% de los usuarios de drogas inyectable informó que en los últimos seis meses no utiliza condón cuando tiene relaciones sexuales con parejas estables; el 54,3\% no lo utilizan con parejas ocasionales y un $22,5 \%$ no lo utilizan con personas que ejercen la prostitución.

Dentro de las características sociodemográficas de los casos positivos con infección de hepatitis C, el 85,7\% eran hombres, el 71,4\% habían iniciado la secundaria, con una edad media de 27,69 años y el $75 \%$ de los usuarios con he-

Tabla 3

Factores de riesgo asociados con hepatitis $C$ en usuarios de drogas inyectables OR crudos y ajustados

\begin{tabular}{|c|c|c|c|c|c|c|c|}
\hline Variable & $\%$ & ORc & IC $95 \%$ & Valor de $p$ & ORa & IC $95 \%$ & Valor de $p$ \\
\hline \multicolumn{8}{|c|}{ Tiempo de inyección } \\
\hline$>4$ años & 26,8 & 1,71 & $1,13-2,57$ & 0,01 & 1,68 & $0,94-3,00$ & 0,08 \\
\hline$<4$ años & 18,1 & & & & & & \\
\hline \multicolumn{8}{|c|}{ Uso del condón } \\
\hline No & 27 & 7,01 & $0,91-54,18$ & 0,06 & 6,63 & $0,83-52,80$ & 0,07 \\
\hline Si & 5 & & & & & & \\
\hline \multicolumn{8}{|c|}{ VIH positivo } \\
\hline $\mathrm{Si}$ & 42,9 & 2,71 & $0,59-12,44$ & 0,20 & 10,22 & $0,84-124,22$ & 0,07 \\
\hline No & 21,7 & & & & & & \\
\hline \multicolumn{8}{|c|}{ Compra jeringas en droguerías (últimos seis meses) } \\
\hline No & 39 & 2,69 & $1,32-5,48$ & 0,01 & 2,96 & $1,09-8,01$ & 0,03 \\
\hline $\mathrm{Si}$ & 19,2 & & & & & & \\
\hline \multicolumn{8}{|l|}{ Sexo } \\
\hline Mujeres & 22,9 & 1,03 & $0,44-2,40$ & 0,95 & 1,43 & $0,52-3,97$ & 0,48 \\
\hline Hombres & 22,4 & & & & & & \\
\hline \multicolumn{8}{|c|}{ Edad X(DS) } \\
\hline $\mathrm{HCV}$ & $26,6(5,5)$ & 1,03 & $0,98-1,08$ & 0,19 & 0,98 & $0,91-1,05$ & 0,65 \\
\hline No HCV & $27,6(6,0)$ & & & & & & \\
\hline
\end{tabular}

Nota. ${ }^{*}$ ORc: razón de disparidad cruda. ${ }^{*}$ ORa: razón de disparidad ajustada. ${ }^{*}$ IC: Intervalos de confianza

Tabla 4. Características de la inyección en usuarios de drogas inyectables y VHC. Armenia 2014.

\begin{tabular}{|c|c|c|}
\hline En los últimos seis meses... & Si & IC $95 \%$ \\
\hline Se ha inyectado con jeringas que tuvieran la mezcla lista para ser usada & $12,7 \%$ & $8,5-16,9$ \\
\hline Ha dividido con otra persona la mezcla & $21,3 \%$ & $16,0-26,6$ \\
\hline Ha compartido con otras personas algodones, cucharas, o agua & $21,7 \%$ & $16,5-26,9$ \\
\hline Ha sacado dosis de una mezcla de droga que otras personas también estaban compartiendo & $19,3 \%$ & $14,3-24,3$ \\
\hline Se ha inyectado en un sitio de consumo al aire libre & $79,9 \%$ & $74,9-85,0$ \\
\hline Alguna vez lo ha inyectado una persona que cobra por inyectar & $21,6 \%$ & $16,4-26,8$ \\
\hline Ha inyectado alguna vez con una jeringa "casera" & $7,4 \%$ & $4,0-10,7$ \\
\hline
\end{tabular}


patitis C tenían 32 años o menos. Dentro de las prácticas de consumo, como sucede con VIH, el haber consumido inyectables durante más de dos años y el compartir material de inyección resultaron tener mayor frecuencia de ocurrencia en los casos positivos como conductas de riesgo previamente documentadas pero que no fueron estadísticamente significativas en esta investigación.

Un importante hallazgo es que las personas que no han conseguido las jeringas en las droguerías en los últimos seis meses tenían 2,7 (IC95\%: 1,32-5,48) veces el riesgo de adquirir la infección por hepatitis $\mathrm{C}$, que los usuarios que reportaron que las habían adquirido en estos lugares.

Con respecto a otras prácticas de riesgo en la inyección, como el compartir algodones, cucharas, agua, no se encontraron diferencias estadísticamente significativas en tener estas prácticas y hepatitis C.

\section{Discusión}

Según ONUSIDA existe suficiente evidencia que demuestra que los programas de reducción de daños pueden disminuir significativamente la transmisión de infecciones como el VIH y VHC entre usuarios de drogas inyectables (UNAIDS, 2012.). Sin embargo, en Colombia esta realidad todavía no se demuestra. Las políticas oficiales sobre drogas siguen prestando la principal atención a la reducción de la oferta y del tráfico de drogas (USAID, 2012) y este es uno de los pocos estudios realizados sobre las prácticas de los usuarios de drogas inyectables en una ciudad intermedia del país (Berbesi et al., 2013), y el único trabajo que incluye la detección de hepatitis $\mathrm{C}$ en UDIs bajo la metodología de RDS (Ramirez et al., 2005). Este estudio identificó un número significativo de usuarios de drogas inyectables que tienen prácticas de riesgo, como compartir jeringas y una baja utilización del condón; factores de riesgo documentados y discutidos ampliamente en otros países desde hace varias décadas (Ball, et al., 1998; Des Jarlais \& Friedman, 1987; Des Jarlais et al., 2013).

En relación con las características sociodemográficas, los UDIs de este estudio, al igual que sucede en el resto del mundo, son predominantemente de sexo masculino, con una media de edad de 26 años inferior a lo reportada en otras investigaciones (Clatts et al., 2010; Platt et al., 2009;). E1 $50 \%$ de los usuarios tenían cuatro años de haber iniciado la inyección; la evidencia disponible sugiere que el virus de la hepatitis $\mathrm{C}$, se adquiere con relativa rapidez después del inicio de la inyección (Garten et al., 2004); aspecto fundamental en la prevención y control de la infección de los usuarios que han iniciado esta práctica de riesgo.

En México, los factores individuales, sociales y ambientales se asociaron independientemente con la infección por VIH entre usuarios de drogas inyectables en Tijuana. Estos hallazgos sugieren la necesidad de intervenir no solamente en los comportamientos de riesgo individuales, sino en los procesos sociales que impulsan estos comportamientos (Strathdee et al., 2008).

La cuarta parte de los UDIs de este estudio, se encuentran infectados por el VIH o VHC, en forma de infección única o de coinfección, cifras muy bajas a las encontradas en otros países (Platt et al., 2008; Ruan et al., 2007). No obstante, este colectivo presenta una alta prevalencia en comparación con la población general del país (Ministerio de Salud y Proteccion Social, 2012), vinculado no sólo al uso de drogas por vía inyectable, sino además a otras características y conductas de riesgo (Hagan et al., 2007).

La transmisión a través de jeringas y agujas compartidas, el haber consumido drogas inyectables por más de cuatro años, y el mantener relaciones sexuales sin utilizar condón, son factores de riesgo clásicos para la adquisición del virus de hepatitis C y VIH (Shapatava et al., 2006); en la presente investigación se identificó seis veces el riesgo de hepatitis C en las personas que no usaron condón en los últimos seis meses.

Con respecto al sexo femenino, en este estudio, no resultó ser una característica de riesgo significativa para las infecciones, a diferencia de lo que observado en otras investigaciones (Oliveira \& Paiva, 2007). Dado que las mujeres se han identificado como propensas a trabajar en la prostitución, a tener relaciones sexuales bajo la influencia de sustancias, y están en una posición de sumisión que no les permite la negociación de sexo seguro, aumentando la transmisión del VIH y convirtiéndose en un problema aún más complicado de salud pública (Mendez et al., 2009).

De otro lado en este trabajo, se identificó como factor de riesgo para hepatitis $\mathrm{C}$, el no adquirir las jeringas en droguerías, lo que posiblemente sugiera la adquisición de jeringas en otros lugares (expendios de drogas, ollas" entre otros), las cuales podrían estar contaminadas, dado que en Colombia no existen programas de intercambio de jeringas. También en esta investigación se halló una tendencia directamente proporcional entre positividad a las infecciones y una mayor frecuencia de inyecciones, similar a lo observado en una investigación realizada en Uruguay (Osimani et al.,2005).

Los esfuerzos exitosos para prevenir la transmisión de infecciones entre los UDI han estado relacionados con la implementación de programas de reducción de daños; sin embargo, ninguna intervención ha dado lugar a la eliminación de comportamientos de riesgo. Para reducir la transmisión del VIH, las estrategias de reducción de daños, pueden ser suficientes, mientras que el control de HCV puede requerir el uso de las prácticas de inyección que garanticen la eliminación de la exposición a equipos contaminados.

Se han reconocido diferencias importantes en la epidemiología del VIH y el VHC en diferentes poblaciones de usuarios de drogas por vía intravenosa. La prevalencia del VIH varía de $5 \%$ a $80 \%$, teniendo un rango menor la pre-

2 Lugar de encuentro utilizado para el microtrafico y consumo de drogas 
valencia del VHC (50-90 \%). Los factores que favorecen la rápida propagación del VHC entre los UDI sugieren que la infección por VHC en una población de usuarios de drogas inyectables puede hacerse endémico en un período de tiempo relativamente corto, siendo mas lenta la transmisión del VIH (Hagan \& Jarlais, 2000; Platt et al., 2009).

Se requieren acciones de manera pronta para impedir una epidemia en Colombia; es muy importante que el país puede dar prioridad a las políticas de reducción de daños y estrategias de prevención de la hepatitis C y el VIH para usuarios de drogas inyectables, mediante la implementación de programas de intercambio de jeringas, así como la eliminación segura de jeringas y agujas contaminadas, entre otros medidas de salud pública.

Como limitaciones de este estudio, se reconoce que el muestreo basado en el encuestado, se considera un muestreo no aleatorio que debe cumplir con una serie de supuestos para ser considerado como una muestra independiente y representativa de la red social; no es posible realizar asociaciones de causa efecto entre la infección por el VHC y los factores de riesgo encontrados debido al tipo de diseño (estudio transversal); la población de estratos más altos no se alcanzó a identificar en este estudio posiblemente por el poco interés en recibir un incentivo por participar. Por último, los datos se obtuvieron a partir de los reportes de las prácticas de riesgo, es posible, que inexactitudes en las respuestas, podría haber afectado los resultados.

En conclusión, se identificó un perfil de usuario de drogas inyectables: hombre, menor de 34 años de edad, con educación secundaria, soltero, con alta frecuencia de inyección, con bajo uso del condón en los últimos seis meses, y una cuarta parte de ellos infectados por el VIH o VHC en forma de infección única o de coinfección; así mismo uno de cada tres comparte jeringas; para Colombia esta investigación constituye un primer paso en la búsqueda de estrategias de prevención.

\section{Reconocimientos}

Investigación financiada por el Ministerio de Justicia y del Derecho

\section{Conflictos de interés}

No se tienen conflictos de interés.

\section{Bibliografía}

Ball, A. L., Rana, S., \& Dehne, K. L. (1998). HIV prevention among injecting drug users: responses in developing and transitional countries. Public Health Reports, 113, 170-181.

Berbesi, D., Montoya, L., \& Segura, A. (2012). Prevalencia de VIH y comportamientos de riesgo en UDI en Medellín y Pereira. Bogotá: Ministerio de Protección Social.
Berbesi, D., Segura, A., \& Montoya, L. (2013). Cross-sectional study of HIV prevalence and the characteristics of injecting drug users in Colombia. Journal of Substance Use, 36, 27-31.

Berbesi-Fernandez, D., Montoya-Vélez L., \& Segura-Cardona A. (2013). Uso del condón y conocimientos sobre prevención del VIH entre los inyectadores de drogas de dos ciudades colombianas. Adicciones, 25, 321-326.

Bravo, MJ., Mejia, IE., \& Barrio, G. (2008). Encuestas de Comportamiento en Consumidores de Drogas Con Alto Riesgo (CODAR): Herramientas Básicas. Washington, D.C.: Organización Panamericana de la Salud

Clatts, M. C., Colón-López, V., Giang, L. M., \& Goldsamt, L. A. (2010). Prevalence and incidence of HCV infection among Vietnam heroin users with recent onset of injection. Journal of Urban Health: Bulletin of the New York Academy of Medicine, 87, 278-291.

Des Jarlais, D. C., \& Friedman, S. R. (1987). HIV infection among intravenous drug users: epidemiology and risk reduction. AIDS, 1, 67-76.

Des Jarlais, D. C., Pinkerton, S., Hagan, H., Guardino, V., Feelemyer, J., Cooper, H. \& Uuskula, A. (2013). 30 Years on Selected Issues in the Prevention of HIV among Persons Who Inject Drugs. Advances in Preventive Medicine, 23, 1-10.

Garten, R. J., Lai, S., Zhang, J., Liu, W., Chen, J., Vlahov, D., \& Yu, X. F. (2004). Rapid transmission of hepatitis C virus among young injecting heroin users in Southern China. International Journal of Epidemiology, 33, 182-188.

Gómez, A. P. (2013). Colombia: fuera del ojo del huracán, pero todavía cerca. Adicciones, , 25, 106-108.

Hagan, H., \& Des Jarlais, D. C. (2000). HIV and HCV infection among injecting drug users. The Mount Sinai Journal of Medicine, 67, 423-428.

Hagan, H., Des Jarlais, D. C., Stern, R., Lelutiu-Weinberger, C., Scheinmann, R., Strauss, S., \& Flom, P. L. (2007). $\mathrm{HCV}$ synthesis project: preliminary analyses of HCV prevalence in relation to age and duration of injection. The International Journal on Drug Policy, 18, 341-351.

Magiorkinis, G., Sypsa, V., Magiorkinis, E., Paraskevis, D., Katsoulidou, A., Belshaw, R., \& Hatzakis, A. (2013). Integrating Phylodynamics and Epidemiology to Estimate Transmission Diversity in Viral Epidemics. PLoS Computational Biology, 9, 1-11.

Mejía, I. E., \& Gómez, A. P. (2005). La inyección de drogas en Bogotá: una amenaza creciente. Adicciones, 17, 251-260.

Mendez Diz, A. M., Schwarz, P., \& Camarotti, A. C. (2009). Prácticas sexuales en usuarios de drogas y riesgo de transmisión del VIH/sida. Rev. argent. sociol, 7, 150-163.

Ministerio de Salud y Protección Social. (2012). Informe mundial de avances en la lucha contra el Sida. Colombia 2012. Recuperado de http://www.unaids.org/en/dataanalysis/knowyourresponse/countryprogressreports/ 2012countries/ 
Oliveira, J. F., \& Paiva, M. S. (2007). Vulnerabilidade de mulheres usuárias de drogas ao HIV/AIDS em uma perspectiva de gênero. Escola Anna Nery, 11, 625-631.

OMS. (2014.). Nota Descriptiva No. 64. Hepatitis C. Recuperado de http://www.who.int/mediacentre/factsheets/fs164/es/

OMS, UNODC, UNAIDS. (2012). Guides for countries to set targets for universal access to HIV prevention, treatment and care for injecting drug users. Recuperado de http:/ /apps.who. int/iris/bitstream/10665/77969/1/9789241504379_ eng.pdf

ONUSIDA. (2012). Datos fundamentales sobre el sida. Recuperado de http://search2.unaids.org/custom/search.asp

ONUSIDA. (2000). Guías sobre la vigilancia del VIH de segunda generacion. Recuperado de http:/ / cedoc.cies.edu.ni/ general/2nd_Generation $\% 20 \% 28 D \% 29 /$ Surveillance $\%$ 20Guidelines/2nd\%20Generation \% 20Surveillance/2nd\%20gen\%20Spa.pdf

Osimani, M., Chiparelli H., \& Latorre L. (2005). Seroprevalencia para los virus de la inmunodeficiencia humana, hepatitis B y C en usuarios de drogas inyectables. Uruguay, 2003. Revista Médica del Uruguay, 21. Recuperado de http://www.scielo.edu.uy/scielo.php?script=sci_arttextypid=S0303

Platt, L., Rhodes, T., Hickman, M., Mikhailova, L., Lisetsky, K., Sarang, A. \& Parry, J. (2008). Changes in HIV prevalence and risk among new injecting drug users in a Russian city of high HIV prevalence. Journal of Acquired Immune Deficiency Syndromes, 47, 623-631.

Platt, L., Vickerman, P., Collumbien, M., Hasan, S., Lalji, N., Mayhew, S., \& Hawkes, S. (2009). Prevalence of HIV, $\mathrm{HCV}$ and sexually transmitted infections among injecting drug users in Rawalpindi and Abbottabad, Pakistan: evidence for an emerging injection-related HIV epidemic. Sexually Transmitted Infections, 85, 17-22.

Ramirez-Valles, J., Heckathorn, D. D., Vázquez, R., Diaz, R. M., \& Campbell, R. T. (2005). From networks to populations: the development and application of respondent-driven sampling among IDUs and Latino gay men. AIDS and Behavior, 9, 387-402.

Ruan, Y., Qin, G., Yin, L., Chen, K., Qian, H.-Z., Hao, C. \& Shao, Y. (2007). Incidence of HIV, hepatitis C and hepatitis $\mathrm{B}$ viruses among injection drug users in southwestern China: a 3-year follow-up study. AIDS, 21, 39-46.

Shapatava, E., Nelson, K. E., Tsertsvadze, T., \& del Rio, C. (2006). Risk behaviors and HIV, hepatitis B, and hepatitis $\mathrm{C}$ seroprevalence among injection drug users in Georgia. Drug and Alcohol Dependence, 82, S35-38.

Strathdee, S. A., Lozada, R., Pollini, R. A., Brouwer, K. C., Mantsios, A., Abramovitz, D. A., \& Patterson, T. L. (2008). Individual, Social, and Environmental Influences Associated With HIV Infection Among Injection Drug Users in Tijuana, Mexico. Journal of Acquired Immune Deficiency Syndromes, 47, 369-376.
UNAIDS. (2012). Global Report. UNAIDS report on the global AIDS epidemic. Recuperado de http://www.unaids.org/

UNAIDS. (2014). Harm reduction works. Recuperado de http://www.unaids.org/es/resources/documents/2014/name,92033,es.asp

USAID. (2012). Uso de drogas y transmisión del VIH en America Latina. AIDSTAR-One. Recuperado de http://www.aidstar-one.com/sites/default/files/AIDSTAR-One_uso_de_ drogas_y_transmision_del_VIH_en_america_latina.pdf

WHO, UNODC, UNAIDS. (2012). Technical guide for countries to set targets for universal access to HIV prevention, treatment and care for injecting drug users. Recuperado de http://www.who.int/hiv/pub/idu/targets_universal_ access/en/ 